\title{
Some structural and numerical aspects of Heisenberg's matrix mechanics with application to one-dimensional nonpolynomial potentials
}

\author{
Ching-Teh $\mathrm{Li}^{*}$ and Gwo-Hong Suen \\ Department of Physics, National Taiwan University, Taipei, Taiwan 10764, Republic of China \\ Abraham Klein ${ }^{\dagger}$ \\ Department of Physics, University of Pennsylvania, Philadelphia, Pennsylvania 19104-6396
}

(Received 4 December 2000; published 18 April 2001)

\begin{abstract}
The equation-of-motion method based on Heisenberg's matrix mechanics, utilized in this paper, was originally developed within the context of the nuclear many-body problem. The method was later applied successfully to anharmonic oscillators and other problems in quantum mechanics with polynomial potentials in one and two dimensions. Here we apply the method to the one-dimensional quantum system with the nonpolynomial potential $\lambda \sinh ^{2} x$, as an illustration of its applicability to a wider class of problems than has been previously considered. We examine some of the structural and numerical aspects of this one-dimensional quantum system, and show that it is essential to work with $\sinh x$ instead of $x$ as the dynamical variable for this nonpolynomial potential problem. This choice is suggested by a preliminary study of the exactly solvable Poschl-Teller potential, $-\lambda / \cosh ^{2} x$, where our methods provide an exact algebraic solution. For the potential $\lambda \sinh ^{2} x$, the same methods yield equations that can be studied numerically by a controlled sequence of nonperturbative approximations.
\end{abstract}

DOI: 10.1103/PhysRevA.63.052110

PACS number(s): 03.65.Ta, 03.65.Ge, 21.60.-n

\section{INTRODUCTION}

The equation-of-motion (EOM) method based on Heisenberg's matrix mechanics utilized in this paper was originally developed to restore the broken symmetries of a mean-field description of nuclear many-body systems [1]. In this method, where exact eigenstates of the Hamiltonian are used, the matrix elements of operators are unknown quantities to be solved from the matrix element equations of the relevant equations of motion and commutation relations. The practical aspects as well as the numerical structure of Heisenberg's matrix mechanics were further expounded [2-4] through applications to various one-dimensional anharmonic oscillator problems. More recently [5-7], we have used the same framework to study the Einstein-Brillouin-Keller (EBK) quantization [8-10] and the associated problem of quantumclassical correspondence for nonseparable systems possessing a phase-space structure of invariant tori.

Here we apply the method to the one-dimensional quantum system with the nonpolynomial potential $\lambda \sinh ^{2} x$. We examine some of the structural and numerical aspects of this one-dimensional quantum system and show that it is essential to work with $\sinh x$ instead of $x$ as the dynamic variable for this nonpolynomial potential problem. The advantages of this choice are illustrated first by application to the exactly solvable Poschl-Teller potential $-\lambda / \cosh ^{2} x$.

Methods quite similar to ours were developed independently by Henkel and Uzes [11], Uzes [12], Halpern [13], and Halpern and Yonkman [14]. These papers deal only with anharmonic oscillators. Among numerous other studies of

\footnotetext{
*Email address: ctli@phys.ntu.edu.tw

${ }^{\dagger}$ Email address: aklein@walet.physics.upenn.edu
}

the anharmonic oscillator based on sum rules and recursion relations, we mention Refs. [15-20].

\section{RECAPITULATION OF THE EQUATION-OF-MOTION METHOD}

In this section, we summarize the essence of the equationof-motion (EOM) method. We consider the one-dimensional quantum system with the Hamiltonian

$$
H=\frac{p^{2}}{2}+V(x),
$$

where $V(x)$ is the potential energy and in the units $\hbar=1$ the position $x$ and momentum operator $p$ satisfy the commutation relation

$$
[x, p]=i .
$$

It is easy to see that Eqs. (2.1) and (2.2) yield the following equations of motion:

$$
\begin{gathered}
{[x, H]=i p,} \\
{[i p, H]=V^{\prime}(x),}
\end{gathered}
$$

where $V^{\prime}(x)=(d / d x) V(x)$ is the derivative of the potential energy. Using Eq. (2.3) to eliminate the momentum operator in favor of the position operator, one can rewrite the equation of motion Eq. (2.4) in the form of a double commutator of $x$ with the Hamiltonian,

$$
[[x, H], H]=V^{\prime}(x) .
$$

Similarly, the commutation relation (2.2) can be put into the form 


$$
[[x, H], x]=1 .
$$

Working in the representation in which $H$ is diagonal with eigenvalues $E_{n}(n=0,1,2, \ldots)$, it is straightforward to obtain from the matrix elements of Eqs. (2.5) and (2.6) between the eigenstates $|n\rangle$ and $\left|n^{\prime}\right\rangle$ of $H$ the expressions

$$
\begin{gathered}
\left(E_{n^{\prime}}-E_{n}\right)^{2} x_{n n^{\prime}}=\left(V^{\prime}\right)_{n n^{\prime}}, \\
\sum_{n^{\prime \prime}}\left(2 E_{n^{\prime \prime}}-E_{n}-E_{n^{\prime}}\right) x_{n n^{\prime \prime}} x_{n^{\prime \prime} n^{\prime}}=\delta_{n n^{\prime}} .
\end{gathered}
$$

In Eqs. (2.7) and (2.8), $x_{n n^{\prime}}=\left\langle n|x| n^{\prime}\right\rangle$ and $\left(V^{\prime}\right)_{n n^{\prime}}$ $=\left\langle n\left|V^{\prime}(x)\right| n^{\prime}\right\rangle$ are, respectively, the matrix elements of the position operator and $V^{\prime}$ between exact eigenstates $|n\rangle$ and $\left|n^{\prime}\right\rangle$. In evaluating the matrix elements $\left(V^{\prime}\right)_{n n^{\prime}}$ in terms of $x_{n n^{\prime}}$ for polynomial potentials, one often has to invoke infinite summations over intermediate states such as that in Eq. (2.8). As has been shown previously [3], one only needs the diagonal matrix elements, i.e., those with $n^{\prime}=n$, in Eq. (2.8), since the off-diagonal elements are not independent of the equations of motion. Therefore, Eq. (2.7) together with Eq. (2.8) with $n^{\prime}=n$ constitute an infinite set of coupled algebraic equations to be solved for the unknowns $x_{n n^{\prime}}$ and $E_{n}$ $-E_{0}$. To facilitate the solution, one depends on the property

$$
\left|x_{n, n \pm 1}\right| \gg\left|x_{n, n \pm 3}\right| \gg\left|x_{n, n \pm 5}\right| \gg \cdots,
$$

valid for symmetric polynomial potentials, which can be used to reduce the infinite set of coupled equations to an approximate finite set. This hierarchy property among the matrix elements $x_{n n^{\prime}}$ can be argued by using the nodal structures of the wave functions with respect to the quantum number $n$ or by using the convergence properties of the infinite intermediate sums such as that in Eq. (2.8) for $n^{\prime}=n$.

The hierarchy property among the matrix elements $x_{n n^{\prime}}$, as given above in Eq. (2.9), implies that the infinite intermediate sums such as that in Eq. (2.8) usually are saturated by just a few intermediate states. It therefore allows one to reduce the infinite set of equations, consisting of Eq. (2.7) with various $n, n^{\prime}$ and Eq. (2.8) with $n^{\prime}=n$, to an approximate finite set to be solved for just a finite number of matrix elements $x_{n n^{\prime}}$ and excitation energies $E_{n}-E_{0}$ at one time. Thus, in the first order of approximation, one keeps only the dominant or fundamental matrix element $x_{n, n \pm 1}$ and neglects all other matrix elements $x_{n, n \pm \nu}$ with $\nu>1$. In the second order of approximation, one then keeps only the matrix elements $x_{n, n \pm 1}$ and $x_{n, n \pm 3}$ and neglects all other matrix elements $x_{n, n \pm \nu}$ with $\nu>3$, and so on. In other words, this hierarchy property allows one to have an order-by-order approximation scheme to solve Eq. (2.7) with various $n, n^{\prime}$ and Eq. (2.8) with $n^{\prime}=n$ for $x_{n n^{\prime}}$ and $E_{n}-E_{0}$. However, we emphasize that this order-by-order approximation scheme is completely nonperturbative in nature because no small coupling parameters are required.

\section{APPLICATION TO THE POSCHL-TELLER POTENTIAL}

In applying the EOM method, as briefly described in the preceding section, to quantum systems with nonpolynomial potentials, there is obviously a problem of how to evaluate $\left(V^{\prime}\right)_{n n^{\prime}}$, the matrix elements of the derivative of the potential, in terms of $x_{n n^{\prime}}$. This problem was encountered in previous studies [3] of several exactly solvable models with nonpolynomial potentials. The simplest such example, which is of interest for the problem studied in this paper, is described by the Hamiltonian with the Poschl-Teller potential,

$$
H=\frac{1}{2} p^{2}-\frac{\lambda}{\cosh ^{2} x},
$$

where the problem is solved elegantly by replacing $x$ by

$$
v \equiv \sinh x
$$

in the fundamental equations of the method. We shall now present a solution of this model that differs in some details from that given previously [3] and also contains some new results.

The equation of motion and commutation relation such as Eqs. (2.5) and (2.6) with $v$ replacing $x$ are given as

$$
[[v, H], H]=-(v H+H v)-\frac{1}{4} v
$$

and

$$
[[v, H], v]=1+v^{2} .
$$

The fact that the double commutator of $v$ with the Hamiltonian, i.e., $[[v, H], H]$, turns out to be linear in $v$ signifies that the Poschl-Teller potential of Eq. (3.1) can be exactly solved in analytic form. Because the Hamiltonian appears on the right-hand side of the equation of motion Eq. (3.3), in a form that does not lead to an energy difference, we will also need one more relation such as

$$
\begin{aligned}
{\left[\left[v^{2}, H\right], H\right]=} & -2\left\{\left(1+2 v^{2}\right) H+H\left(1+2 v^{2}\right)\right\} \\
& -2\left(1+2 v^{2}\right)-4 \lambda,
\end{aligned}
$$

referred to as the generalized virial theorem.

In the present EOM method, it is essential to work in the representation in which $H$ is diagonal, $H|n\rangle=E_{n}|n\rangle$, as the basis states. Thus, by taking the matrix elements of the equation of motion Eq. (3.3) between the exact eigenstates $|n\rangle$ and $\left|n^{\prime}\right\rangle$, one obtains

$$
\left\{\left(E_{n^{\prime}}-E_{n}\right)^{2}+E_{n}+E_{n^{\prime}}+\frac{1}{4}\right\} v_{n n^{\prime}}=0,
$$

where $v_{n n^{\prime}} \equiv\left\langle n|v| n^{\prime}\right\rangle$. Similar to the case of a harmonic oscillator, except that $x$ has been replaced by $v$, Eq. (3.6) implies that the only nonvanishing matrix elements $v_{n n^{\prime}}$ are those with $n^{\prime}=n \pm 1$. This equation yields for $n^{\prime}=n+1$,

$$
W_{n}^{2}+W_{n}+2 E_{n}+\frac{1}{4}=0,
$$


where the notation $W_{n} \equiv E_{n+1}-E_{n}$ has been used. On the other hand, the diagonal matrix elements of Eqs. (3.4) and (3.5) in the states $|n\rangle$ yield, respectively,

$$
\left[W_{n}-\frac{1}{2}\right] v_{n, n+1}^{2}-\left[W_{n-1}+\frac{1}{2}\right] v_{n-1, n}^{2}=\frac{1}{2}
$$

and

$$
\left(2 E_{n}+1\right)\left\{1+2\left(v_{n, n+1}^{2}+v_{n-1, n}^{2}\right)\right\}+2 \lambda=0 .
$$

Here, $v_{n, n^{\prime}}^{2} \equiv\left(v_{n n^{\prime}}\right)^{2}$ and the property that $v_{n n^{\prime}}=v_{n^{\prime} n}$ has been used. Noting that $v_{n-1, n}=0$ for $n=0$, Eqs. (3.7)-(3.9) with $n=0$ readily yield

$$
\begin{gathered}
W_{0}=\sqrt{2 \lambda+\frac{1}{4}}-1, \\
E_{0}=-\frac{1}{2}\left[\sqrt{2 \lambda+\frac{1}{4}}-\frac{1}{2}\right]^{2}, \\
v_{01}=\sqrt{\frac{1}{2 W_{0}-1}} .
\end{gathered}
$$

From Eq. (3.7), we have directly

$$
W_{n} \equiv E_{n+1}-E_{n}=-\frac{1}{2}+\sqrt{-2 E_{n}},
$$

and by comparing the same equation for $n$ and $n+1$, we can conclude that

$$
W_{n}=W_{n-1}-1 .
$$

Noting that $E_{n}=W_{n-1}+W_{n-2}+\cdots+W_{0}+E_{0}$, it is not difficult to use Eqs. (3.11) and (3.14) to derive a general result for the bound-state energies, which takes the form

$$
E_{n}=-\frac{1}{2}\left[\sqrt{2 \lambda+\frac{1}{4}}-\left(n+\frac{1}{2}\right)\right]^{2},
$$

where $n=0,1,2, \ldots, N$ with $N$ being an integer smaller than but closest to $\sqrt{2 \lambda+1 / 4}$. Thus, for the Poschl-Teller potential in Eq. (3.1), there are only a finite number of bound states with energies given by Eq. (3.15), and the continuum starts at $E=0$.

Finally, we derive a result that was not given in Ref. [3], a general formula for the nonvanishing matrix elements of $v$, i.e., $v_{n, n \pm 1}$. Although Eq. (3.8) provides a recursive relation for evaluating $v_{n, n+1}$, it seems to be only useful for numerical evaluations of $v_{n, n \pm 1}$. In any event, we have not found an analytic solution for the general $v_{n, n+1}$ using this equation alone. Thus, instead of solving Eq. (3.8) recursively, we solve for fixed $n$ the two quantities $v_{n-1, n}^{2}$ and $v_{n, n+1}^{2}$ directly from both Eqs. (3.8) and (3.9) in terms of the known results for $E_{n}$ and $W_{n}$. Then, after employing relations such as that in Eq. (3.14) and some further manipulations, it is not difficult to get the following general formula for the nonvanishing matrix elements:

$$
v_{n, n+1}=\sqrt{\frac{(n+1)\left(2 W_{0}-n+1\right)}{\left(2 W_{0}-2 n-1\right)\left(2 W_{0}-2 n+1\right)}} .
$$

From Eq. (3.10), it is easy to see that for large enough $\lambda$ and small quantum number $n$, one has $W_{0} \gg n$ and

$$
v_{n, n+1} \rightarrow x_{n, n+1} \approx \sqrt{\frac{n+1}{2 \sqrt{2 \lambda}}} .
$$

This strong-coupling limit for low-lying states is consistent with the fact that the lowest-order term $\lambda x^{2}$ in the powerseries expansion of the potential (with an overall coupling constant $\lambda$ ) dominates for large $\lambda$. We emphasize that formulas like Eq. (3.16) for matrix elements $v_{n, n+1}$ are not easy to obtain with other quantum-mechanical methods not based on Heisenberg's matrix mechanics.

\section{APPLICATION TO OTHER NONPOLYNOMIAL POTENTIALS}

In the application of the EOM method to the PoschlTeller potential in the preceding section, we have seen that the problem of evaluating the matrix element of $V^{\prime}$, the derivative of the potential, in terms of $x_{n n^{\prime}}$ can be avoided by working with $\sinh x$ instead of $x$ as the dynamical variable. Because the Poschl-Teller potential is exactly solvable, it is worthwhile to apply the present method to other nonpolynomial potentials which must be solved numerically. Part of the purpose of this further application is to illustrate the applicability of the method to a wider class of problems than has been previously considered. As shown in the preceding section, for nonpolynomial potentials it is essential to replace the position operator $x$ with some appropriate function of $x$, say $f(x)$, as the dynamical variable $[3,4]$. Thus, instead of Eqs. (2.5) and (2.6), one uses the following equation of motion and commutation relation in the form of double commutators for $f(x)$ [4]. Namely,

$$
[[f, H], H]=-\left(f^{\prime \prime} H+H f^{\prime \prime}\right)+2 f^{\prime \prime} V+f^{\prime} V^{\prime}-\frac{1}{4} f^{(4)}
$$

and

$$
[[f, H], f]=\left(f^{\prime}\right)^{2},
$$

where $f^{\prime}, f^{\prime \prime}$, and $f^{(4)}$ are the first, second, and fourth derivatives of $f(x)$ with respect to $x$, respectively. As we have already seen for the Poschl-Teller potential, we shall also need the double-commutator equation of motion for $f^{2}(x)$.

Here, for illustration, we specifically apply the EOM method to the following quantum system with a nonpolynomial potential $V(x)=\lambda \sinh ^{2} x$, where the coupling constant $\lambda>0$. Thus, the Hamiltonian is given by

$$
H=\frac{p^{2}}{2}+\lambda \sinh ^{2} x .
$$

Choosing $v \equiv \sinh x$ as the dynamical variable, Eqs. (4.1) and (4.2) with $f(x)=v$ become

$$
[[v, H], H]=-(v H+H v)+\left(2 \lambda-\frac{1}{4}\right) v+4 \lambda v^{3},
$$

and 


$$
[[v, H], v]=1+v^{2} .
$$

Besides appearing in the commutators, the Hamiltonian itself also appears in the right-hand side of the equation of motion above. We thus need an extra relation. As before, we use the double commutator of $v^{2}$ with the Hamiltonian, i.e., Eq. (4.1) with $f=v^{2}$, which gives

$$
\begin{aligned}
{\left[\left[v^{2}, H\right], H\right]=} & -2\left\{\left(1+2 v^{2}\right) H+H\left(1+2 v^{2}\right)\right\} \\
& +12 \lambda v^{4}+(8 \lambda-4) v^{2}-2 .
\end{aligned}
$$

Working in the basis formed by the exact eigenstates $|n\rangle$ of $H$, i.e., $H|n\rangle=E_{n}|n\rangle$ with $n=0,1,2, \ldots$, we obtain the matrix elements of Eq. (4.4) between the $|n\rangle$ and $\left|n^{\prime}\right\rangle$ as

$$
\left[\left(E_{n^{\prime}}-E_{n}\right)^{2}+E_{n}+E_{n^{\prime}}-2 \lambda+\frac{1}{4}\right] v_{n n^{\prime}}-4 \lambda\left(v^{3}\right)_{n n^{\prime}}=0,
$$

the diagonal matrix elements of Eq. (4.5) as

$$
\sum_{n_{1}}\left(E_{n_{1}}-E_{n}-\frac{1}{2}\right) v_{n n_{1}} v_{n_{1} n}=\frac{1}{2},
$$

and the diagonal matrix elements of Eq. (4.6) as

$$
-\left[1+2\left(v^{2}\right)_{n n}\right] E_{n}+3 \lambda\left(v^{4}\right)_{n n}+(2 \lambda-1)\left(v^{2}\right)_{n n}-\frac{1}{2}=0,
$$

where $\left(v^{2}\right)_{n n}$ and $\left(v^{4}\right)_{n n}$ can be evaluated by the sum rules such as

$$
\left(v^{4}\right)_{n n}=\sum_{n_{1}, n_{2}, n_{3}} v_{n n_{1}} v_{n_{1} n_{2}} v_{n_{2} n_{3}} v_{n_{3} n} .
$$

If one expands the potential energy $\lambda \sinh ^{2} x$ as a power series in $x$, it can be shown that the contribution of the lowest-power terms, especially the $x^{2}$ term, dominates numerically for large $\lambda$. On this basis and also for the infinite sum such as that in Eq. (4.8) to converge, it is reasonable to expect that the matrix elements of $v$ should also have the property such as that in Eq. (2.9), namely,

$$
\left|v_{n, n \pm 1}\right| \gg\left|v_{n, n \pm 3}\right| \gg\left|v_{n, n \pm 5}\right| \gg \cdots .
$$

This assumption is validated by our numerical results. This hierarchy property among the matrix elements $v_{n n^{\prime}}$ implies that the infinite intermediate sums such as those in Eq. (4.10) usually are saturated by just a few states. It therefore allows one to reduce the infinite set of equations, consisting of Eqs. (4.7)-(4.9), to an approximate finite set to be solved for just a finite number of matrix elements $v_{n n^{\prime}}$ and energies $E_{n}$ at one time. Thus, in the first order of approximation, one keeps only the dominant matrix element $v_{n, n \pm 1}$ and neglects all other matrix elements $v_{n, n \pm \nu}$ with $\nu>1$. In the second order of approximation, one keeps only the matrix elements $v_{n, n \pm 1}$ and $v_{n, n \pm 3}$ and neglects all other matrix elements $v_{n, n \pm \nu}$ with $\nu>3$, and so on. In other words, the hierarchy property given in Eq. (4.11) allows one to have an order-by-order approximation scheme to solve Eqs. (4.7)-(4.9) for $v_{n n^{\prime}}$ and $E_{n}$. Again we emphasize that this order-by-order approxima- tion scheme is completely nonperturbative in nature because no small parameters are required.

\section{A. First-order calculations}

We consider the first-order approximation in which one keeps only the matrix elements between neighboring exact eigenstates, i.e., $v_{n, n \pm 1}$, and neglects all other matrix elements $v_{n, n \pm \nu}$ with $\nu>1$. Noting that $v_{n n^{\prime}}=v_{n^{\prime} n}$, Eqs. (4.7) - (4.9) with $n^{\prime}=n+1$ yield

$$
\begin{gathered}
W_{n}^{2}+W_{n}+2 E_{n}-4 \lambda\left[v_{n-1, n}^{2}+v_{n, n+1}^{2}\right. \\
\left.+v_{n+1, n+2}^{2}\right]-2 \lambda+\frac{1}{4}=0, \\
{\left[W_{n}-\frac{1}{2}\right] v_{n, n+1}^{2}-\left[W_{n-1}+\frac{1}{2}\right] v_{n-1, n}^{2}=\frac{1}{2},} \\
3 \lambda\left[v_{n, n+1}^{4}+v_{n, n+1}^{2} v_{n+1, n+2}^{2}+2 v_{n-1, n}^{2} v_{n, n+1}^{2}\right. \\
\left.+v_{n-1, n}^{2} v_{n-2, n-1}^{2}+v_{n-1, n}^{4}\right]+(2 \lambda-1)\left[v_{n, n+1}^{2}+v_{n-1, n}^{2}\right] \\
-\frac{1}{2}-\left[1+2\left(v_{n, n+1}^{2}+v_{n-1, n}^{2}\right)\right] E_{n}=0,
\end{gathered}
$$

where $W_{n} \equiv E_{n+1}-E_{n}, v_{n, n^{\prime}}^{2}=\left(v_{n, n^{\prime}}\right)^{2}$, and $v_{n, n^{\prime}}^{4}=\left(v_{n, n^{\prime}}\right)^{4}$, etc. Also, $n=0,1,2, \ldots, N$ with $N$ being the quantum number of the highest state in the first-order calculation. To make Eqs. (4.12)-(4.14) a closed set of equations, we need a closure relation expressing $v_{N, N+1}$ in terms of $v_{N-1, N}$. One such relation is provided by Eq. (4.15) below. Alternatively, for large enough $N$, it is reasonable to set $v_{N+1, N+2}$ $=v_{N, N+1}$. The particular choice makes little difference in the numerical results for low-lying states. After such an approximation has been made, Eqs. (4.12)-(4.14) then constitute a set of $3(N+1)$ coupled equations to be solved for $v_{n, n+1}$, $W_{n}$, and $E_{n}$ with $n=0,1,2, \ldots, N$. Numerical results obtained this way will be labeled as $\mathrm{C} 1$, meaning coupled firstorder calculations, in the various tables below.

As mentioned above, the series expansion for the potential energy $\lambda \sinh ^{2} x$ is dominated by the lower-power terms, especially the $x^{2}$ term, for large coupling constant $\lambda$. Treating the $x^{4}$ and all other higher-power terms as perturbations to the harmonic $x^{2}$ term, it can be shown that for any $n$, up to the first order of the perturbative calculations, one has the relationships

$$
v_{n, n+1}=\sqrt{\frac{n+1}{n}} v_{n-1, n}
$$

and

$$
W_{n-1}=W_{n}-\frac{1}{2} .
$$

Although these relationships have been derived in the strongcoupling limit, we shall investigate the consequences of using them for all values of $\lambda$. Thus, using Eq. (4.15) to express $v_{n+1, n+2}$ and $v_{n-1, n}$ in terms of $v_{n, n+1}$ and Eq. (4.16) to express $W_{n-1}$ in terms of $W_{n}$, Eqs. (4.12)-(4.14) for different $n$ can be decoupled to yield, respectively,

$$
W_{n}^{2}+W_{n}+2 E_{n}-12 \lambda v_{n, n+1}^{2}-2 \lambda+\frac{1}{4}=0,
$$


TABLE I. Comparison of the uncoupled and coupled first-order results, denoted, respectively, by $\mathrm{U} 1$ and $\mathrm{C} 1$, with exact values for $H=\frac{1}{2} p^{2}+\lambda \sinh ^{2} x$ with $\lambda=50$.

\begin{tabular}{cccc}
\hline \hline & $\mathrm{U} 1$ & $\mathrm{C} 1$ & Exact \\
\hline$E_{0}$ & 5.102 & 5.099 & 5.1221 \\
$E_{1}$ & 15.55 & 15.56 & 15.5999 \\
$E_{2}$ & 26.45 & 26.45 & 26.5314 \\
$E_{3}$ & 37.81 & 37.75 & 37.8918 \\
$E_{4}$ & 49.60 & 49.43 & 49.6606 \\
$E_{5}$ & 61.83 & 61.49 & 61.820 \\
$v_{01}$ & 0.2241 & 0.2241 & 0.2236 \\
$v_{12}$ & 0.3181 & 0.3179 & 0.3164 \\
$v_{23}$ & 0.3915 & 0.3911 & 0.3878 \\
$v_{34}$ & 0.4547 & 0.4539 & 0.4482 \\
$v_{45}$ & 0.5117 & 0.5102 & 0.5017 \\
$v_{03}$ & 0.00606 & 0.00609 & 0.00612 \\
$v_{14}$ & 0.01163 & 0.01162 & 0.01167 \\
$v_{05}$ & 0.00025 & 0.00024 & 0.000270 \\
\hline \hline
\end{tabular}

$$
\begin{gathered}
\left(W_{n}-\frac{n+1}{2}\right) v_{n, n+1}^{2}=\frac{n+1}{2} \\
3 \lambda \frac{6 n^{2}+6 n+3}{(n+1)^{2}} v_{n, n+1}^{4}+(2 \lambda-1) \frac{2 n+1}{n+1} v_{n, n+1}^{2}-\frac{1}{2} \\
-\left(\frac{4 n+2}{n+1} v_{n, n+1}^{2}+1\right) E_{n}=0 .
\end{gathered}
$$

For each $n$, one only has to solve these three equations for the three unknowns $v_{n, n+1}, W_{n}$, and $E_{n}$. The numerical results so obtained are labeled as U1, meaning uncoupled first-order calculations, in the various tables below. The energies $E_{n}$ obtained directly from solving the three equations above are less accurate than the results $\mathrm{C} 1$, except for the lowest couple of states. It is more accurate to calculate the energies of the excited states by adding the various $W_{n}$ to the ground-state energy $E_{0}$. Or, alternatively, after the various $v_{n, n+1}$ with different $n$ have been obtained from Eqs. (4.17)-(4.19), one can evaluate the energies using Eq. (4.14), which can be rewritten as

$$
\begin{aligned}
E_{n}= & \left\{3 \lambda \left[v_{n, n+1}^{4}+v_{n, n+1}^{2} v_{n+1, n+2}^{2}+2 v_{n-1, n}^{2} v_{n, n+1}^{2}\right.\right. \\
& \left.+v_{n-1, n}^{2} v_{n-2, n-1}^{2}+v_{n-1, n}^{4}\right]+(2 \lambda-1)\left[v_{n, n+1}^{2}+v_{n-1, n}^{2}\right] \\
& \left.-\frac{1}{2}\right\} /\left[1+2\left(v_{n, n+1}^{2}+v_{n-1, n}^{2}\right)\right] .
\end{aligned}
$$

All the energies given in Tables I-IV in the U1 columns have been evaluated this way using $v_{n, n+1}$ and $W_{n}$ obtained from solving Eqs. (4.17)-(4.19). On the other hand, those excited-state energies given in the $\mathrm{C} 1$ columns have been evaluated by adding the various $W_{n}=E_{n+1}-E_{n}$ to the ground-state energy $E_{0}$. Should they have been calculated in exactly the same way, the energies given in both the U1 and $\mathrm{C} 1$ columns in Tables I-IV would have been much closer to each other.
TABLE II. Comparison of the uncoupled and coupled first-order results, denoted, respectively, by $\mathrm{U} 1$ and $\mathrm{C} 1$, with exact values for $H=\frac{1}{2} p^{2}+\lambda \sinh ^{2} x$ with $\lambda=10$.

\begin{tabular}{cccc}
\hline \hline & $\mathrm{U} 1$ & $\mathrm{C} 1$ & Exact \\
\hline$E_{0}$ & 2.321 & 2.309 & 2.3550 \\
$E_{1}$ & 7.214 & 7.212 & 7.2831 \\
$E_{2}$ & 12.58 & 12.52 & 12.625 \\
$E_{3}$ & 18.40 & 18.20 & 18.343 \\
$E_{4}$ & 24.69 & 24.23 & 24.409 \\
$E_{5}$ & 31.48 & 30.62 & 30.80 \\
$v_{01}$ & 0.3375 & 0.3370 & 0.3343 \\
$v_{12}$ & 0.4827 & 0.4813 & 0.4739 \\
$v_{23}$ & 0.6002 & 0.5969 & 0.5821 \\
$v_{34}$ & 0.7049 & 0.6986 & 0.6742 \\
$v_{45}$ & 0.8022 & 0.7918 & 0.7562 \\
$v_{03}$ & 0.01859 & 0.01856 & 0.01830 \\
$v_{14}$ & 0.03429 & 0.03440 & 0.03366 \\
$v_{05}$ & 0.00134 & 0.00140 & 0.00147 \\
\hline \hline
\end{tabular}

Before we conclude these first-order calculations, we comment on how the matrix elements of $x$ and $p$ between the exact eigenstates of the Hamiltonian can be evaluated. For this, one has to go back to Eq. (2.7) or Eq. (2.8). In the first order of approximation by keeping only $x_{n, n \pm 1}$, Eq. (2.8) with $n^{\prime}=n$ yields [3]

$$
\left(E_{n+1}-E_{n}\right) x_{n, n+1}^{2}=\frac{n+1}{2} .
$$

Thus, this together with Eq. (2.3) imply that

$$
x_{n, n+1}=x_{n+1, n}=\sqrt{\frac{n+1}{2 W_{n}}},
$$

TABLE III. Comparison of the uncoupled and coupled firstorder results, denoted, respectively, by $\mathrm{U} 1$ and $\mathrm{C} 1$, with exact values for $H=\frac{1}{2} p^{2}+\lambda \sinh ^{2} x$ with $\lambda=5$.

\begin{tabular}{lccc}
\hline \hline & $\mathrm{U} 1$ & $\mathrm{C} 1$ & Exact \\
\hline$E_{0}$ & 1.655 & 1.638 & 1.6980 \\
$E_{1}$ & 5.232 & 5.219 & 5.3027 \\
$E_{2}$ & 9.289 & 9.195 & 9.2987 \\
$E_{3}$ & 13.83 & 13.53 & 13.643 \\
$E_{4}$ & 18.87 & 18.21 & 18.306 \\
$E_{5}$ & 24.45 & 23.23 & 23.26 \\
$v_{01}$ & 0.4039 & 0.4028 & 0.3974 \\
$v_{12}$ & 0.5815 & 0.5783 & 0.5645 \\
$v_{23}$ & 0.7291 & 0.7216 & 0.6946 \\
$v_{34}$ & 0.8636 & 0.8495 & 0.8058 \\
$v_{45}$ & 0.9911 & 0.9680 & 0.9052 \\
$v_{03}$ & 0.0296 & 0.0296 & 0.02867 \\
$v_{14}$ & 0.0540 & 0.0539 & 0.05177 \\
$v_{05}$ & 0.0028 & 0.0028 & 0.00289 \\
\hline \hline
\end{tabular}


TABLE IV. Comparison of the uncoupled and coupled firstorder results, denoted, respectively, by $\mathrm{U} 1$ and $\mathrm{C} 1$, with exact values for $H=\frac{1}{2} p^{2}+\lambda \sinh ^{2} x$ with $\lambda=1$.

\begin{tabular}{cccc}
\hline \hline & $\mathrm{U} 1$ & $\mathrm{C} 1$ & Exact \\
\hline$E_{0}$ & 0.745 & 0.7051 & 0.8169 \\
$E_{1}$ & 2.58 & 2.522 & 2.6313 \\
$E_{2}$ & 4.96 & 4.714 & 4.7753 \\
$E_{3}$ & 7.91 & 7.241 & 7.199 \\
$E_{4}$ & 11.5 & 10.10 & 9.87 \\
$E_{5}$ & 15.7 & 13.3 & 12.7 \\
$v_{01}$ & 0.6221 & 0.6160 & 0.5942 \\
$v_{12}$ & 0.9210 & 0.9032 & 0.8519 \\
$v_{23}$ & 1.196 & 1.153 & 1.0554 \\
$v_{34}$ & 1.465 & 1.384 & 1.2317 \\
$v_{45}$ & 1.734 & 1.61 & 1.39 \\
$v_{03}$ & 0.0824 & 0.0822 & 0.0762 \\
$v_{14}$ & 0.1412 & 0.1427 & 0.1318 \\
$v_{05}$ & 0.0111 & 0.0122 & 0.0120 \\
\hline \hline
\end{tabular}

$$
p_{n, n+1}=-p_{n+1, n}=-i \sqrt{\frac{(n+1) W_{n}}{2}}
$$

The energy eigenvalues are then given approximately by

$$
E_{n}=\frac{1}{2}\left\{\frac{(n+1) W_{n}}{2}+\frac{n W_{n-1}}{2}\right\}+\lambda\left[v_{n, n+1}^{2}+v_{n-1, n}^{2}\right] .
$$

Energy eigenvalues evaluated this way can be used as a consistency check against those given in Tables I-IV evaluated using Eqs. (4.20). Numerically, both procedures give approximately the same accuracy except for systems with small $\lambda$ or for states with high quantum numbers $n$.

Overall, the present first-order calculations yield very accurate results for large $\lambda$ and states with low $n$. For smaller $\lambda$ and states with higher $n$, these results are less accurate but still reasonable. To get better results for these cases, one has to perform higher-order calculations by keeping some of the subdominant matrix elements that have been neglected so far.

\section{B. Evaluation of some subdominant matrix elements}

The first-order calculations illuminated above not only yield approximately the energies $E_{n}$ and the fundamental matrix elements $v_{n, n \pm 1}$, but they also allow one to evaluate the subdominant matrix elements such as $v_{n, n \pm 3}, v_{n, n \pm 5}$, and so on.

To evaluate these subdominant matrix elements, we employ the off-diagonal matrix elements of the commutation relation given in Eq. (4.5). Namely, with $n^{\prime} \neq n$, one has

$$
\sum_{n_{1}}\left(2 E_{n_{1}}-E_{n}-E_{n^{\prime}}-1\right) v_{n, n_{1}} v_{n_{1}, n^{\prime}}=0
$$

Setting $n^{\prime}=n+2$ and keeping only $v_{n . n \pm 1}$ and $v_{n, n \pm 3}$, Eq. (4.25) yields

$$
\begin{aligned}
v_{n, n+3}= & {\left[\left(E_{n}+E_{n+2}-2 E_{n+1}+1\right) v_{n, n+1} v_{n+1, n+2}\right.} \\
& \left.-\left(E_{n}+E_{n+2}-2 E_{n-1}+1\right) v_{n-1, n} v_{n-1, n+2}\right] / \\
& {\left[\left(2 E_{n+3}-E_{n}-E_{n+2}-1\right) v_{n+2, n+3}\right] . }
\end{aligned}
$$

Starting with $n=0$, this recursive relation allows one to evaluate $v_{03}, v_{14}$, and so on. Similarly, setting $n^{\prime}=n+4$ and keeping only $v_{n, n \pm \nu}$ with $\nu \leqslant 5$ in Eq. (4.25), one can readily obtain a similar recursive relation for evaluating $v_{05}$, $v_{16}$, and so on. Numerical results for some of these subdominant matrix elements together with the fundamental matrix elements $v_{n, n+1}$ are given in the lower parts of Tables I-IV below.

\section{Higher-order calculations}

After the first-order calculations have been done, one may proceed to do higher-order calculations by keeping more and more subdominant matrix elements $v_{n n^{\prime}}$ according to the hierarchy given in Eq. (4.11). In actual calculations, however, it is more convenient to choose a subspace consisting of the exact eigenstates $|n\rangle$ of the Hamiltonian with $n$ $=0,1,2, \ldots, N$ and keep all matrix element $v_{n n^{\prime}}$ within this subspace. One then solves for these matrix elements and energies within this subspace from Eqs. (4.7)-(4.9). Note that one only uses Eq. (4.8) with $n=0,1,2, \ldots, N-1$. Results obtained this way are obviously more accurate for quantities involving lower-lying states and less accurate for quantities involving higher-lying states. Apparently, here we do not have a consistent order of approximation based on Eq. (4.11) for all the states in the chosen subspace. However, the present scheme is easier to implement numerically.

By increasing the value of $N$, i.e., increasing the number of states in the subspace, one can get more and more accurate results for both the matrix elements $v_{n n^{\prime}}$ and energies $E_{n}$. When the subspace chosen is large enough, it is seen that the energies of low-lying states and the corresponding matrix elements no longer change within a preset numerical accuracy and thus can be considered exact. The larger $N$ is chosen, the larger the set of exact results found. The "exact" energies and matrix elements given in Tables I-IV have been obtained this way.

\section{CONCLUDING REMARKS}

In this paper, we first briefly described the essence of the equation-of-motion (EOM) method in the framework of Heisenberg matrix mechanics. To show that the EOM method has applicability to a wider class of problems than previously considered, we applied it to one-dimensional quantum systems with nonpolynomial potentials. As a first illustration, we studied the quantum system with the PoschlTeller potential, which is exactly solvable. There we showed that it was essential to work with $\sinh x$ in place of the position operator $x$ as the dynamical variable. For other nonpolynomial potentials, we stress that it is also important to work with an appropriate function of the position operator as the dynamical variable. Thus, instead of the equations of motion and commutation relations for the position operator, one 
works with those for the chosen function of the position operator. By specifically applying the EOM method to the quantum system with the potential $\lambda \sinh ^{2} x$, which must be solved numerically, we have shown that the present method provides one with an order-by-order but nonperturbative calculation scheme for evaluating not only the energy eigenvalues but also the various matrix elements of interest. It should be clear that the example chosen, $\lambda \sinh ^{2} x$, is but one member of a large class of models to which a similar analysis can be applied. For example, our method can be easily applied to the double sinh-Gordon potential, which is known to be a quasiexactly solvable model [21]. It will be interesting to examine how this property of a potential manifests itself in the framework of Heisenberg's matrix mechanics. This is currently under investigation and the results will be reported elsewhere.

\section{ACKNOWLEDGMENTS}

We are grateful to J.-M. Yuan and M. Vallières for helpful discussions. This work was supported in part by the ROC National Science Council, through Contract No. NSC892112-M-002-042.
[1] A.K. Kerman and A. Klein, Phys. Lett. 1, 185 (1962).

[2] C.T. Li, A. Klein, and F. Krejs, Phys. Rev. D 17, 2311 (1975).

[3] C.T. Li and A. Klein, Fizika (Zagreb) 22(1), 67 (1990).

[4] C.T. Li, Chin. J. Phys. (Taipei) 32, 309 (1994).

[5] W.R. Greenberg, A. Klein, and C.T. Li, Phys. Rev. Lett. 64, 1244 (1995).

[6] W.R. Greenberg, A. Klein, and C.T. Li, Phys. Rep. 264, 167 (1996).

[7] W.R. Greenberg, A. Klein, I. Zlatev, and C.T. Li, Phys. Rev. A 54, 1820 (1996).

[8] H. Hasegawa, M. Robnik, and G. Wunner, Prog. Theor. Phys. Suppl. 98, 198 (1989).

[9] J. Müller, J. Burgdörfer, and D. Noid, Phys. Rev. A 45, 1471 (1992).

[10] G.S. Ezra, C.C. Martens, and L.E. Fried, J. Phys. Chem. 91,
3721 (1987).

[11] J.H. Henkel and C.A. Uzes, Phys. Rev. D 8, 4430 (1973).

[12] C.A. Uzes, Phys. Rev. D 14, 3362 (1976).

[13] F.R. Halpern, J. Math. Phys. 14, 219 (1973).

[14] F.R. Halpern and T.W. Yonkman, J. Math. Phys. 15, 1718 (1974).

[15] R.J. Swenson and S.H. Danforth, J. Chem. Phys. 57, 1734 (1971).

[16] F.T. Hioe and E.W. Montroll, J. Math. Phys. 16, 1945 (1975).

[17] K. Banarjee, Phys. Lett. 63A, 223 (1977).

[18] J.B. Bronzan and R.L. Sugar, Phys. Rev. D 23, 1806 (1981).

[19] R.H. Tipping and J.F. Ogilvie, Phys. Rev. A 27, 95 (1983).

[20] K. Yamazaki, Prog. Theor. Phys. 70, 629 (1983).

[21] A. Khare and B.P. Mandal, J. Math. Phys. 39, 3476 (1998). 